\title{
Optimal beam forming in the presence of mutual coupling
}

\author{
Roald Goossens, Hendrik Rogier \\ Ghent University, Department of Information Technology, Ghent, Belgium
}

\begin{abstract}
An optimal beam forming method is proposed which incorporates all electromagnetic characteristics of the antenna array, including mutual coupling. The optimal beam forming technique minimizes the total power radiated by the antenna array while the response in a desired direction is maintained. By considering the active element patterns and the impedance matrix a computational efficient beam forming algorithm is obtained which is able to manage all mutual coupling effects. Simulation results show the performance of the proposed algorithm applied to an antenna array in the presence of a platform.
\end{abstract}

\section{INTRODUCTION}

The demand for wireless mobile communication services is growing at an explosive rate. The large number of mobile users and the increasing data rate for applications such as mobile internet require a high performance antenna system. Smart antenna technology, making use of adaptive antenna arrays, is capable of improving system capacity and signal quality, and will be an essential component in third- and fourth-generation cellular systems. A huge amount of research has already been performed on the topic of smart antenna applications, such as beam forming strategies and direction-of-arrival (DOA) estimation.

There is a tendency to develop more compact antenna arrays with a larger number of antenna elements. Because of this the antennas are located closer to each other and the effects of mutual coupling become more significant. Mutual coupling is due to the fact that when an antenna is radiating, some of the energy in one antenna is coupled into a neighboring antenna. In combination with scattering effects from the antenna tower and nearby structures the radiation characteristics of an antenna in such an antenna array can differ significantly from the stand-alone antenna characteristics. High performance smart antenna applications require a correct description of the electromagnetic characteristics including mutual coupling. In this paper we propose a beam forming strategy that incorporates all mutual coupling effects relying on the active element patterns and the impedance matrix. In [1], [2] a coupling matrix is introduced to compensate for mutual coupling effects. The coupling matrix transforms the radiation patterns of the individual antenna elements such that the modified antenna patterns no longer suffer from mutual coupling effects, allowing to apply traditional beam forming algorithms. A disadvantage of this method is that the coupling matrix depends on the elevation angle in the presence of platform effects [2]. Due to this limitation constructing a radiation pattern with constraints in different elevation angles is not possible. In [3] the use of genetic algorithms in beam forming for an array in the presence of a closeby mounting tower is investigated. All mutual coupling effects are taken into account by considering the active element patterns. The active element pattern of an antenna is the radiation pattern of this antenna when the antenna is excited with a unit voltage [4]. In the meanwhile, the voltage at the other ports remains zero and the internal impedance remains fixed at all ports. This leads to a set of active element patterns. Any beam formed by the considered antenna array is a linear combination of the active element patterns. A genetic algorithm searches a linear combination that fits best to the desired specifications. However, in real-time applications less time-consuming algorithms are desirable.

We propose an optimal beam forming scheme [5] that includes all mutual coupling effects such as platform effects, which occur usually at cellular base stations [3]. We search for the array weights that minimize the total power radiated by the antenna array while the power in the direction of the mobile user is maintained. In the up-link communication data are collected which enable us to estimate the direction-of-arrival (DOA) of the mobile users. Several algorithms, based on MUSIC, are developed to estimate the DOAs in the presence of mutual coupling [6]. To incorporate all mutual coupling effects we rely on the exact active element patterns of the antennas. Besides this set of active element patterns, the electromagnetic characteristics of the antenna array can be described by an equivalent circuit model. Whereas a stand-alone antenna possesses a characteristic antenna radiation impedance, an antenna array is described by an impedance matrix. The relation, derived in Section II, between the impedance matrix and the energy in the active element patterns enables us to develop an optimal beam forming strategy. This algorithm is applicable for any array configuration even in the presence of severe mutual coupling, because the algorithm relies on the active element patterns and the impedance matrix.

This paper is organized as follows. In Section II, an expression for the power radiated by an antenna array is derived. We make use of the equivalent circuit model which describes the antenna array by means of the impedance matrix. On the basis of this expression we develop the optimal beam forming algorithm in Section III. We make a distinction between the situations where interferers are either absent or present. In Section IV the proposed beam forming algorithm is illustrated by means of some 
examples. Attention is devoted to antenna configurations with very large mutual coupling effects.

\section{Circuit MODEL VS ANTENNA PATTERN}

Consider an antenna array consisting of $K$ antenna elements. No limitations are imposed on the geometry of the array, nor it is necessary to assume identical antenna elements. The antenna elements of the compact antenna array are situated in the near field of the other antenna elements and an amount of the energy transmitted by one antenna element will be received by the other elements. These elements, for their part, will act as radiating antenna elements. Besides neighboring antenna elements we also consider the presence of nearby scatterers, such as the feed lines of the antenna elements and a mounting platform [3]. Because of the neighboring antennas and the nearby scatterers the radiation pattern of an excited antenna element will differ significantly from the radiation pattern of the identical excited antenna element in a standalone environment. To include all these mutual coupling effects it is possible to consider the detailed current distribution on all the antennas and the nearby scatterers, e.g. determined by a method of moments. In this paper, we go a step further and opt to remove all near field information by considering the active element pattern and the mutual coupling matrix. This compact description still enables us to include all mutual coupling effects.

A single antenna element in transmit mode can be described by its radiation pattern and an equivalent circuit model determined by the antenna input impedance. This approach is extendable to an antenna array of $K$ elements. Now, the antenna input impedance is replaced by a mutual coupling matrix $\mathbf{Z}$, which describes all mutual coupling effects. In Fig. 1 the circuit model for a two-element antenna array in transmit mode is given. Both antennas are excited with a voltage source with an internal impedance $Z_{0}$. The circuit model can easily be extended to $K$ antenna elements. The model is fully described by the internal impedance $Z_{0}$ and the impedance matrix $\mathbf{Z}$ :

$$
[\mathbf{Z}]_{i j}=Z_{i j}
$$

The elements of the impedance matrix can easily be found by exciting antenna element $t$ with a $1 A$ current source and by measuring the voltages at the other antennas which are open circuited. The diagonal elements of the impedance matrix differ from the antenna input impedance of the stand-alone antenna elements, due to the presence of neighboring antennas and nearby scatterers. The offdiagonal elements of the impedance matrix describe the voltages that currents in an antenna element induce in the terminals of the other antenna elements.

The beam pattern, formed by the antenna array, can be controlled by steering the amplitude and the phase of the voltage source at each antenna port. To incorporate all mutual coupling effects we make use of the active element patterns [4]. The active element pattern of an antenna element is obtained by exciting the antenna with a unit voltage, while the other terminals are terminated

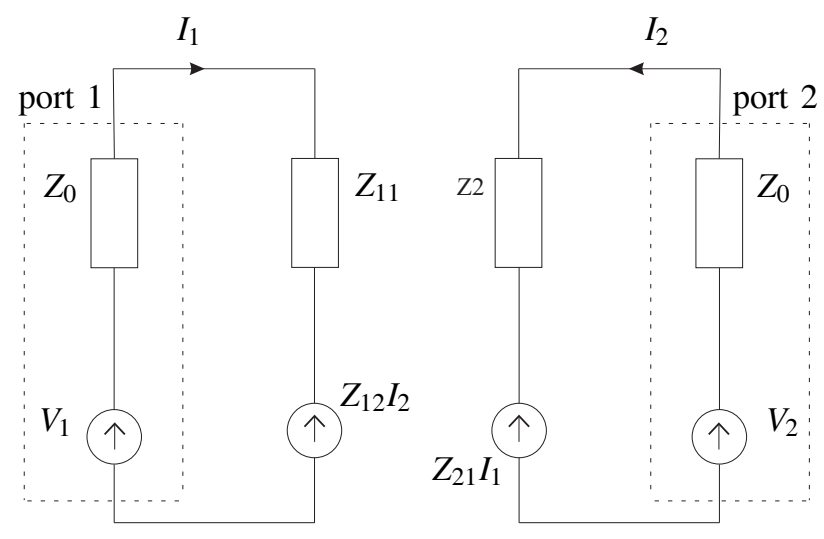

Fig. 1. Circuit model for a two-element antenna array in transmit mode.

by an impedance $Z_{0}(=50 \Omega)$. For an antenna array with $K$ antennas this leads to $K$ unit-excitation active element patterns $\left\{F_{i}(\theta, \varphi)\right\}(i=1 \ldots K)$. For clearness we assume that all antenna elements are uniformly polarized, so that the active element patterns can be treated as scalar functions of the elevation and azimuth angle $(\theta, \varphi)$. However, the approach proposed in this paper is also applicable for diversely polarized antennas. All effects of mutual coupling are incorporated in the concept of the active element pattern. When an antenna element is excited by a voltage source, the impedance matrix induces currents in the other antenna elements. However, the active element pattern of this antenna is not just a simple combination of the stand-alone radiation patterns of all antenna elements. Shadowing effects, induced by nearby scatterers, can deform the radiation patterns. Beside the impedance matrix $\mathbf{Z}$, the $K$ active element patterns are necessary to describe the electromagnetic characteristics of the antenna array in full detail. By a linear combination of the active element patterns $\left\{F_{i}(\theta, \varphi)\right\}$, desired beams can be synthesized.

When the antenna elements are excited with voltages $\left\{V_{i}\right\}(i=1 \ldots K)$ the radiation pattern of the array is

$$
F_{t o t}(\theta, \varphi)=\sum_{i=1}^{K} V_{i} F_{i}(\theta, \varphi)=\mathbf{v f}(\theta, \varphi) .
$$

$\mathbf{f}(\theta, \varphi)$ is a $K \times 1$ column matrix vector which contains the $K$ active element patterns of the array,

$$
\mathbf{f}(\theta, \varphi)=\left[F_{1}(\theta, \varphi), F_{2}(\theta, \varphi), \ldots, F_{K}(\theta, \varphi)\right]^{T} .
$$

Whereas $\mathbf{v}$ is a $1 \times K$ column matrix vector containing the voltages applied at the antenna elements.

The total power radiated by the antenna array is an important characteristic in beam forming algorithms and is given by

$$
P_{\text {rad }}=\frac{1}{2 R_{c}} \iint F_{\text {tot }}(\theta, \varphi) F_{\text {tot }}^{*}(\theta, \varphi) \mathrm{d} \Omega,
$$

where $R_{c}$ is the impedance of the medium in which the antenna radiates and ..* denotes the complex conjugate. 
Substituting (2) in (4) gives:

$$
\begin{gathered}
P_{\text {rad }}=\frac{1}{2 R_{c}} \mathbf{v} \iint \mathbf{f}(\theta, \varphi) \mathbf{f}^{H}(\theta, \varphi) \mathrm{d} \Omega \mathbf{v}^{H} \\
=\mathbf{v} \mathbf{R} \mathbf{v}^{H},
\end{gathered}
$$

.${ }^{H}$ denotes the hermitian of a vector. The matrix $\mathbf{R}$ expresses the correlation between the different active element patterns:

$$
\mathbf{R}=\frac{1}{2 R_{C}} \iint \mathbf{f}(\theta, \varphi) \mathbf{f}^{H}(\theta, \varphi) \mathrm{d} \Omega .
$$

It is obvious that the off-diagonal elements are the result of mutual coupling between the different antenna elements. In absence of mutual coupling, the correlation matrix $\mathbf{R}$ is reduced to a diagonal matrix. When we consider the circuit model, the power inserted in the circuit model is given by the power supplied by the $K$ voltage sources:

$$
P_{\text {input }}=\frac{1}{2} \Re\left(\sum_{i=1}^{K} V_{i} I_{i}^{*}\right)=\frac{1}{2} \Re\left(\mathbf{v i}^{H}\right),
$$

where $\mathbf{i}$ is a $1 \times K$ column matrix vector containing the $K$ corresponding currents in the antenna terminals. The following relationship exists between the excited voltages and the currents in the antenna elements:

$$
\mathbf{v}=\mathbf{i}\left(Z_{0} \mathbf{I}+\mathbf{Z}\right) .
$$

Substituting (9) into (8) yields

$$
P_{\text {input }}=\frac{1}{2} \Re\left(\mathbf{i}\left(Z_{0} \mathbf{I}+\mathbf{Z}\right) \mathbf{i}^{H}\right),
$$

where $\mathbf{I}$ is the $K \times K$ identity matrix. Notice that part of the input power $P_{\text {input }}$ is not used to radiate but is dissipated in the internal impedances of the ports. The energy used to radiate is then given by:

$$
P_{\text {rad }}^{\prime}=P_{\text {input }}-\frac{1}{2} \Re\left(\sum_{i=1}^{K} Z_{0} I_{i} I_{i}^{*}\right)=\frac{1}{2} \Re\left(\mathbf{i} \mathbf{Z} \mathbf{i}^{H}\right) .
$$

We replace $\mathbf{i}$ by $\mathbf{v}\left(Z_{0} \mathbf{I}+\mathbf{Z}\right)^{-1}$ in (11) given (9), which results in:

$$
P_{\text {rad }}^{\prime}=\frac{1}{2} \mathbf{v} \mathfrak{R}\left(\left(Z_{0} \mathbf{I}+\mathbf{Z}\right)^{-1} \mathbf{Z}\left(Z_{0}^{*} \mathbf{I}+\mathbf{Z}^{H}\right)^{-1}\right) \mathbf{v}^{H} .
$$

Both approaches (active element pattern $P_{r a d}$ and circuit model $P_{r a d}^{\prime}$ ) yield an expression for the radiated power. Identifying (4) and (12) relates the active element patterns to the circuit model parameters:

$$
\mathbf{R}=\left(Z_{0} \mathbf{I}+\mathbf{Z}\right)^{-1} \Re(\mathbf{Z})\left(Z_{0}^{*} \mathbf{I}+\mathbf{Z}^{H}\right)^{-1} .
$$

In Section III we rely on this relationship to perform beam steering in an efficient way.

\section{OPTIMAL BEAM FORMING}

Several algorithms were developed to estimate the direction of arrival angles of impinging signals in the uplink of the wireless communication system. To yield accurate estimates mutual coupling effects have to be incorporated into the algorithm [6]. In the down link, the knowledge of the DOAs is used to cancel interference effects between different users by beam forming. First, we demonstrate in section III-A that taking mutual coupling into account does not complicate the optimal beam forming algorithm [5]. Quite the contrary, optimal beam forming is inextricably interconnected with the impedance matrix $\mathbf{Z}$. Second, in section III-B the optimal beam forming algorithm is extended to the case where interference is mitigated by placing nulls in the radiation pattern.

\section{A. Absence of other mobile users}

In the uplink communication it is important to determine the excitation voltages in such way that most of the radiated power is transmitted to a desired user. Optimal beam forming satisfies this condition given that excitation voltages are determined that minimize the power radiated by the array while the power in the direction of the mobile user is maintained. Assume that the desired mobile user is located along the direction $\left(\theta_{0}, \varphi_{0}\right)$. The optimal beam forming algorithm, including mutual coupling, proceeds by searching for the excitation voltages $\left\{V_{i}\right\}$ that fulfill the conditions:

$$
\left\{\begin{array}{l}
\mathbf{v f}\left(\theta_{0}, \varphi_{0}\right)=1 \\
\mathbf{v}^{H} \mathbf{R} \mathbf{v} \text { is minimized. }
\end{array}\right.
$$

The beam forming algorithm does not consider the directions-of-arrival (DOA) of the signals of other mobile users by placing nulls in the radiation pattern in the corresponding directions, but instead it globally minimizes the interference plus noise. Therefore, no tracking of moving interferers is required, we only need to track the changing DOA of the desired user. The solution of this minimization problem can be found by using a Lagrange multiplier. The Lagrangian of the problem becomes

$$
L(\mathbf{v}, \lambda)=\mathbf{v R} \mathbf{v}^{H}+\lambda(\mathbf{v f}-1)
$$

and the solution is

$$
\mathbf{v}_{0}^{H}=\frac{\mathbf{R}^{-1} \mathbf{f}\left(\theta_{0}, \varphi_{0}\right)}{\mathbf{f}\left(\theta_{0}, \varphi_{0}\right)^{H} \mathbf{R}^{-1} \mathbf{f}\left(\theta_{0}, \varphi_{0}\right)} .
$$

Equation (16) expresses that the excitation voltages to obtain an optimal beam in the direction $\left(\theta_{0}, \varphi_{0}\right)$ are determined by the correlation matrix $\mathbf{R}$ and the radiation pattern in the direction $\left(\theta_{0}, \varphi_{0}\right)$ of all active element patterns. In Section II it is proven that the correlation matrix $\mathbf{R}$ is described by the impedance matrix $\mathbf{Z}$. So the optimal beam forming algorithm is linked by the mutual coupling parameters, e.g. the impedance matrix.

\section{B. Interference cancellation}

In the previous section a steering vector is determined that minimizes the total power radiated by the antenna array whereas in a given direction the radiated power is ensured. The presence of other mobile users is disregarded and no precautions are taken to mitigate interference. In some situations it is useful to deform the radiation pattern in such way that nulls are placed in the direction of undesirable mobile users (null steering) [5]. The algorithm 
described in section III-A can easily be extended such that nulls are created. Assume $N$ directions, where the first direction is the direction of the desired mobile user and the other $N-1$ directions determine the directions of the interfering mobile users. We define the matrix $\mathcal{F}$ where the $i$ th column is the collection of all active element patterns in the direction $\left(\theta_{i}, \varphi_{i}\right)$ :

$$
\mathcal{F}=\left[\mathbf{f}\left(\theta_{0}, \varphi_{0}\right), \mathbf{f}\left(\theta_{1}, \varphi_{1}\right), \ldots, \mathbf{f}\left(\theta_{N-1}, \varphi_{N-1}\right)\right] .
$$

In the $1 \times N$ column matrix vector $\boldsymbol{\alpha}$ the wanted response for the total radiation pattern is stored,

$$
\boldsymbol{\alpha}=[1,0, \ldots, 0] .
$$

The constrained optimization algorithm with nulls along the directions of the interferers is described by

$$
\left\{\begin{array}{l}
\mathbf{v} \mathcal{F}=\boldsymbol{\alpha} \\
\mathbf{v}^{H} \mathbf{R} \mathbf{v} \text { is minimized. }
\end{array}\right.
$$

The Lagrangian of this problem becomes

$$
L(\mathbf{v}, \boldsymbol{\lambda})=\mathbf{v R} \mathbf{v}^{H}+(\mathbf{v} \mathcal{F}-\boldsymbol{\alpha}) \boldsymbol{\lambda},
$$

where the Lagrange multiplier is now a $N \times 1$ row vector $\lambda=\left[\lambda_{1}, \ldots, \lambda_{N}\right]^{T}$. The solution of this combined null steering and optimal beam forming algorithm is:

$$
\mathbf{v}^{H}=\mathbf{R}^{-1} \mathcal{F}\left(\mathcal{F}^{H} \mathbf{R}^{-1} \mathcal{F}\right)^{-1} \boldsymbol{\alpha}^{H} .
$$

Although the expression is more complex than (16), the excitation voltages are again determined by the impedance matrix $\mathbf{Z}$ and the collection of the active element patterns. It is obvious that the power radiated by the antenna array in case of extra null conditions is higher than in the case were a single optimal beam is obtained (we assume that the main beam is constructed at the same direction $\left.\left(\theta_{0}, \varphi_{0}\right)\right)$

$$
\mathbf{v} \mathbf{R} \mathbf{v}^{H} \geq \mathbf{v}_{0} \mathbf{R} \mathbf{v}_{0}^{H}
$$

To compare both optimization problems we scale the last problem with a factor such that the total radiated power is the same for both problems. The scaled solution becomes

$$
\mathbf{v}^{H}=\sqrt{\frac{\mathbf{v}_{0} \mathbf{R} \mathbf{v}_{0}^{H}}{\mathbf{v} \mathbf{R} \mathbf{v}^{H}}} \mathbf{R}^{-1} \mathcal{F}\left(\mathcal{F}^{H} \mathbf{R}^{-1} \mathcal{F}\right)^{-1} \boldsymbol{\alpha}^{H} .
$$

Because of this scaling the total radiation pattern in the desired direction will be lower than the imposed unit response.

\section{EXAMPLES AND RESULTS}

We demonstrate the optimal beam forming strategy by means of some well-chosen examples relying on synthetic antenna radiation data generated by means of the well-established NEC2-code. However, the beam forming algorithm is generally applicable even in more realistic and practical antenna array systems. Consider a uniform circular array consisting of nine dipole antenna elements (see Fig. 2) tuned to $900 \mathrm{MHz}$ (dipole length $l=\frac{\lambda}{2}=$ $16.12 \mathrm{~cm})$. The array elements are distributed uniformly on a circle with diameter $d=l\left(\approx \frac{\lambda}{2}\right)$. In the center of the circle there is a short-circuited dipole with length

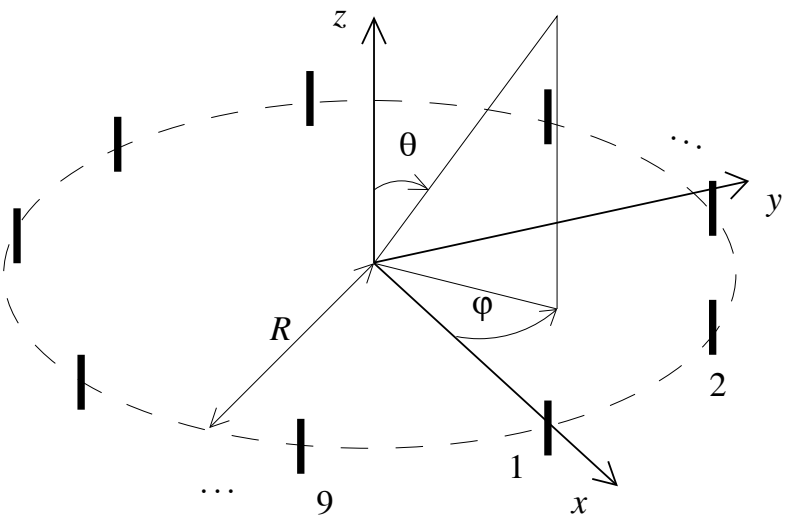

Fig. 2. Uniform circular array composed of nine dipole antenna elements.

$l=16.12 \mathrm{~cm}$. The choice for an additional short-circuited dipole in the center of the array is well-considered, because in real-life applications antenna arrays are mounted on a platform and the short-circuited dipole acts like a mast of the platform. To visualize the mutual coupling effects, the active element pattern of the dipole element 1 situated on the $x$-axis is shown. It is clear that this active element pattern differs from the omnidirectional radiation pattern of the stand-alone dipole. The presence of a nearby scatterer (short-circuited dipole) is also important because we see in Fig. 3 that the active element pattern of the same dipole element without the short-circuited dipole in the center is different.

The result of mutual coupling introduced by neighboring scatterers and nearby scatterers is that traditional beam forming strategies no longer deliver satisfactory beam patterns because of the deformation of the active element patterns. In Fig. 4 the beam patterns are shown

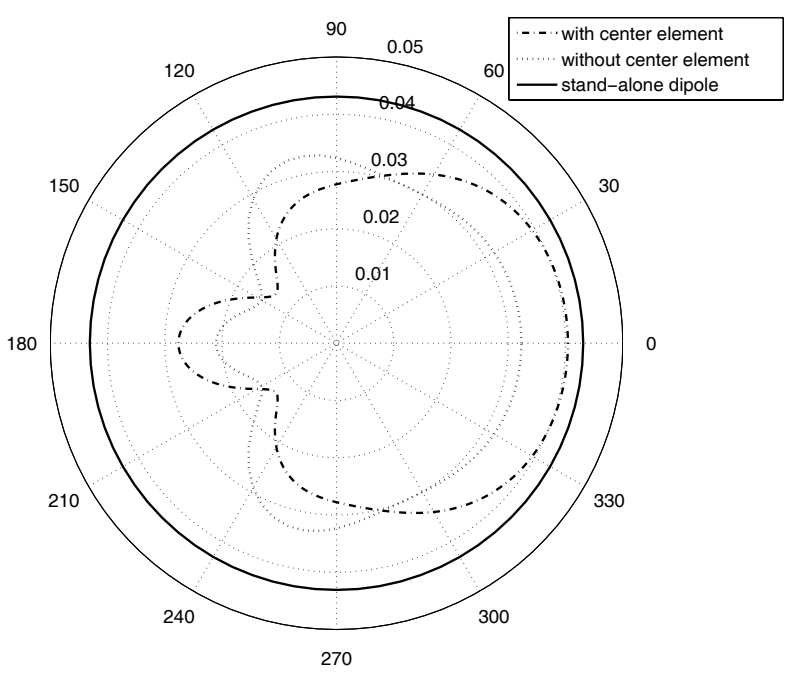

Fig. 3. The active element patterns of the dipole antenna in three different electromagnetic neighborhoods. 
for three different strategies of beam forming in the direction $\left(90^{\circ}, 110^{\circ}\right)$. In a first case a phase compensation is applied assuming that the radiation pattern is not affected by mutual coupling. This phase compensation yield a radiation pattern with unit response in the desired direction when the omnidirectional radiation pattern is considered. Mutual coupling deforms the radiation pattern of the individual elements and we see in Fig. 4 that the unit response is no longer achieved. A maximum in the radiation pattern in the desired direction is still obtained, but the destructive interference in the other regions is insufficiently for creating small side-lobes. Second, a phase compensation is applied assuming the exact active element patterns. This elementary beam forming strategy results in complete constructive interference in the desired direction and destructive interference in the other regions. This was not clearly manifested in the beam pattern obtained by the first method. The final strategy is the optimal beam forming strategy proposed in this paper. It is the only of the three methods which minimizes the radiated power. This is distinctly reflected in the beam pattern, a smaller beamwidth of the main lobe and smaller side-lobes are the result of the optimal beam forming. It cannot be emphasized enough that the complexity for calculating the excitation voltages by the optimal beam forming is not higher than the other traditional phase compensation techniques. Because the algorithm relies on

(a)

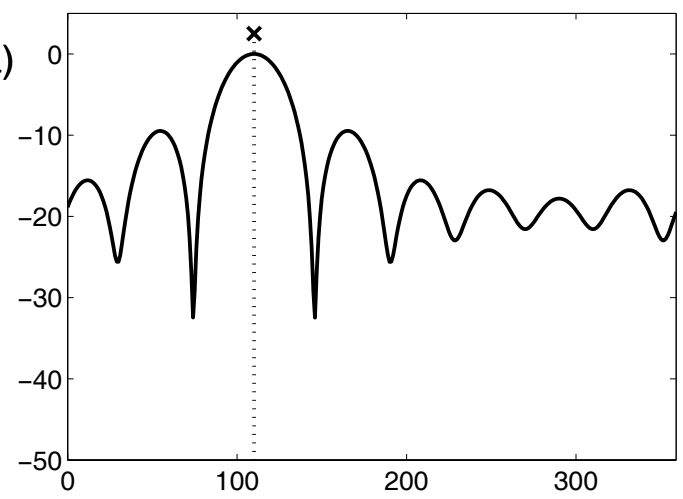

(c)

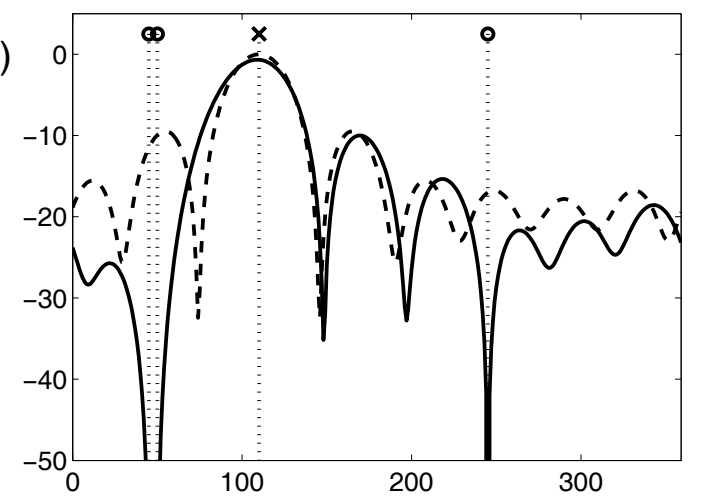

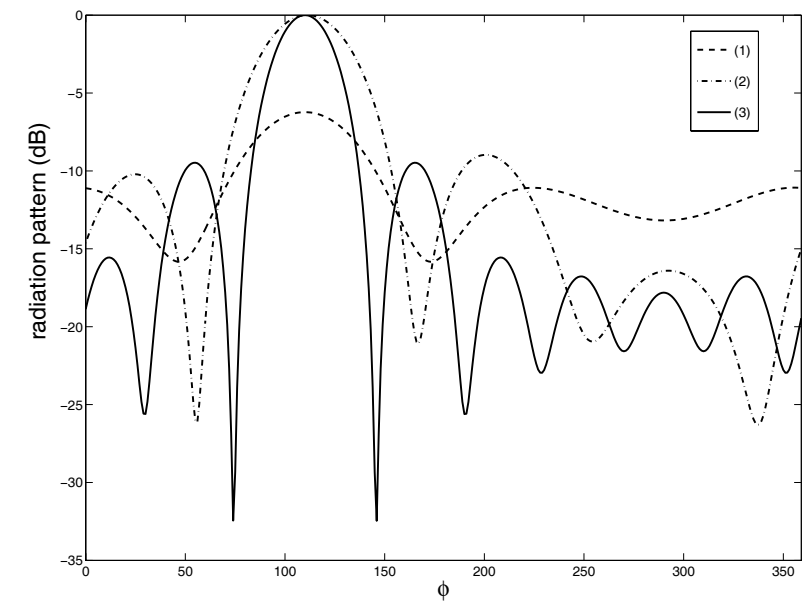

Fig. 4. The radiation pattern obtained by: (1) phase compensation while mutual coupling is neglected, (2) phase compensation when mutual coupling is incorporated, (3) optimal beam forming algorithm.

the electromagnetic properties of the antenna array, such as the active element pattern and the impedance matrix, it can handle all mutual coupling effects.

To demonstrate the optimal beam forming algorithm combined with null steering we consider again the uniform circular array with a short-circuited dipole in the center of the antenna array. We consider four situations.

(b)

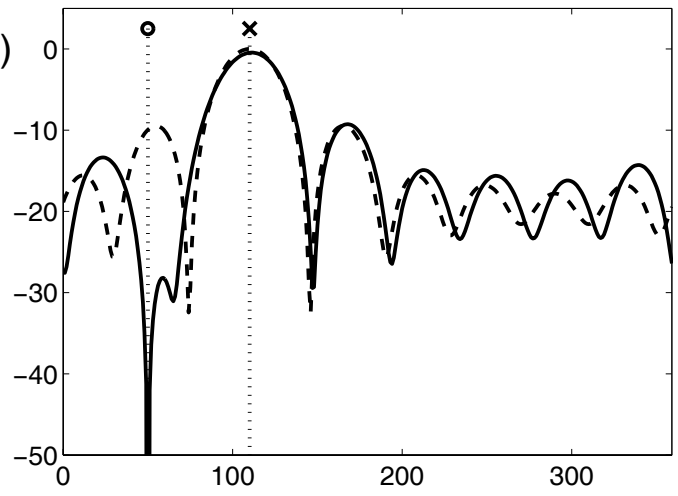

(d)

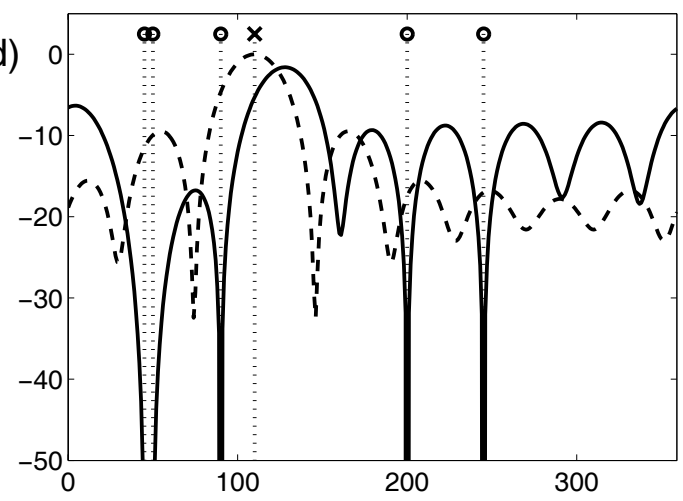

Fig. 5. Comparison of optimal beam forming result at $\varphi=110^{\circ}$ in four situations: (a) absence of interferers, (b) one interferer, (c) three interferers, (d) five interferers. 
TABLE I

THE AZIMUTH ANGLES OF THE PRESENT INTERFERERS

\begin{tabular}{|c|c|}
\hline & directions of the interferers \\
\hline situation 1 & none \\
situation 2 & $50^{\circ}$ \\
situation 3 & $45^{\circ}, 50^{\circ}, 245^{\circ}$ \\
situation 4 & $45^{\circ}, 50^{\circ}, 90^{\circ}, 200^{\circ}, 245^{\circ}$ \\
\hline
\end{tabular}

In all situations we want to steer a beam to the desired mobile user in the direction $\left(90^{\circ}, 110^{\circ}\right)$. In Table I the directions of the different interferers are shown and we assume that all mobile users are situated along the same elevation angle $\theta=90^{\circ}$. In Fig. 5 the radiation patterns are plotted in the four different situations. The radiation pattern obtained in the first situation, where no additional nulls are present, is taken as a reference situation and is included in each subplot. We observe that the radiation pattern is correctly modified such that nulls are present in the direction of the interferers. As a result of these additional nulls the unit response in the desired direction is not maintained, because the radiation pattern is scaled in such way that the total power radiated by the antenna array is the same as in the reference situation. In the third situation, when three interferers are present, we notice that the beam width of the main lobe increases. This shouldn't cause any surprise because imposing extra null constraints interferes with the requirement of minimizing the total radiated power. In the fourth situation the direction of the main lobe differs significantly from the desired direction whereas in the other situations the main lobe was correctly directed to the desired mobile user. The cause of this failure can be attributed to the interferer at the direction $\varphi=90^{\circ}$ which is located too closely to the desired direction. This can be mended by considering a larger antenna array which has an intrinsically smaller beam width.

In the previous examples the desired mobile user and the interferers are all situated in the same azimuth plane $\left(\theta=90^{\circ}\right)$. However, this is not a requirement for the algorithm. We assume that three mobile users are present in the beam forming problem. A desired user is located in the direction $\left(90^{\circ}, 180^{\circ}\right)$, while the other two interferers are located in the directions $\left(80^{\circ}, 230^{\circ}\right)$ and $\left(90^{\circ}, 250^{\circ}\right)$. In Fig. 6 a limited region of the three dimensional radiation pattern is shown. The three specified directions are marked and we notice that at the correct directions nulls are placed and optimal radiation properties are obtained. We also observe that the beamwidth in the azimuth angle is much smaller than in the elevation angle as a result of the circular antenna array configuration. In Fig. 6 the direction $\left(100^{\circ}, 230^{\circ}\right)$ is marked with a dashed line, because at that direction another null is formed although no constraint is imposed in that direction. Yet, the symmetry of the dipole elements results in a radiation pattern which is symmetric around $\theta=90^{\circ}$.

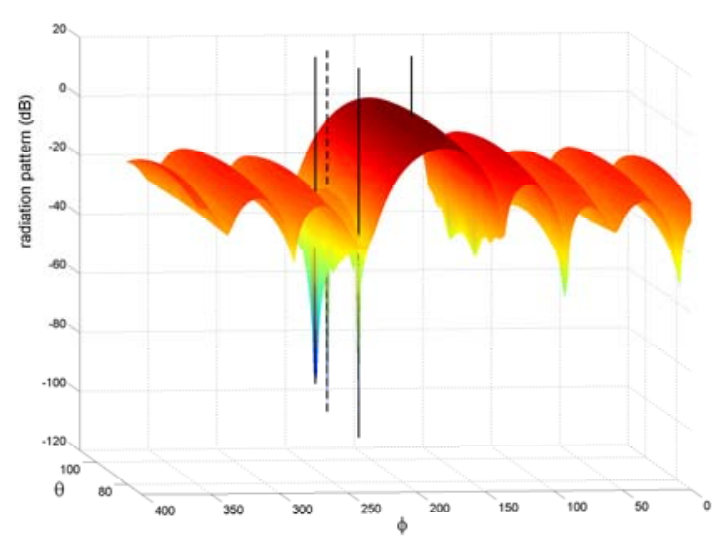

Fig. 6. Comparison of optimal beam forming.

\section{CONCLUSIONS}

Mutual coupling in antenna arrays is always present in whatever degree. We demonstrated that in beam forming applications this has not to be a disadvantage. Instead of compensating mutual coupling we proposed an optimal beam forming algorithm which relies on the active element patterns and the impedance matrix. Because of this the total power radiated by the antenna array is minimized while in certain directions constraints are imposed. The performance of the proposed algorithm is demonstrated by means of an antenna array that suffers severely by mutual coupling.

\section{REFERENCES}

[1] E.B. Perri and L.C. Trintinalia, "Downlink radiation pattern in adaptive array with mutual coupling", Proc. of the Conference Antennas and Propagation Society Int. Symposium, 2004, Vol. 3, pp.2659-2662.

[2] K.R. Dandekar, H. Ling and G. Xu, "Experimental study of mutual coupling compensation in smart antenna applications", IEEE Transactions on wireless communications, Vol.1, no.3 July 2002, pp.480-487.

[3] T. Su and H. Ling, "Array beamforming in the presence of a mounting tower using genetic algorithms", IEEE Trans. on Antennas Propag., Vol.53, no.6, June 2005, pp.2011-2019.

[4] H. Rogier and D. De Zutter, "Beamforming strategies for compact arrays in mobile terminals using the exact active element pattern method", Microwave and Optical Technology Letters, Vol.35, no.3, Nov. 2002, pp.201-203.

[5] L.C. Godara, "Application of antenna arrays to mobile communications, part II: beam-forming and direction-of-arrival considerations", Proc. of the IEEE, Vol.85, no.8, August 1997, pp.11951245.

[6] R. Goossens and H. Rogier, "2-D direction of arrival estimation combining UCA-RARE and MUSIC for uniform circular arrays subject to mutual coupling", Proc. of International Conference on electromagnetics in advanced applications, Sept. 2005 pp.:791 794. 


\section{Symposium on Communications and Vehicular Technology}

Copyright and Reprint Permission: Abstracting is permitted with credit to the source. Libraries are permitted to photocopy beyond the limits of U.S. copyright law for private use of patrons, those articles in this volume that carry a code at the bottom of the first page, provided that the per-copy fee indicated in the code is paid through the Copyright Clearance Center, 222 Rosewood Drive, Danvers, MA 01923. Other copying, reprint, or republication requests should be addressed to the IEEE Copyrights Manager, IEEE Service Center, 445 Hoes Lane, P.O. Box 1331, Piscataway, NJ 08855-1331. All rights reserved. Copyright (C) 2006 by The Institute of Electrical and Electronics Engineers, Inc.

(C) 2006 IEEE. Personal use of this material is permitted. However, permission to reprint/republish this material for advertising or promotional purposes or for creating new collective works for resale or redistribution to servers or lists, or to reuse any copyrighted component of this work in other works must be obtained from the IEEE.

IEEE Catalog Number: 06EX1465C

ISBN: $\quad 0-7803-9785-1$

Library of Congress Number: 2006928412

A few additional copies of this publication are available from:

Mrs M. Delville

AIM

Rue Saint-Gilles, 31

B-4000 Liège

Belgium

+324222 2946

+324222 2388 (FAX)

email: m.delville@aim.skynet.be 


\title{
Proceedings \\ 2006 Symposium on Communications and Vehicular Technology
}

\author{
IEEE SCVT 2006
}

\section{$13^{\text {th }}$ Annual Symposium on Communications and Vehicular Technology in the Benelux}

November 23, 2006

Liège, Belgium
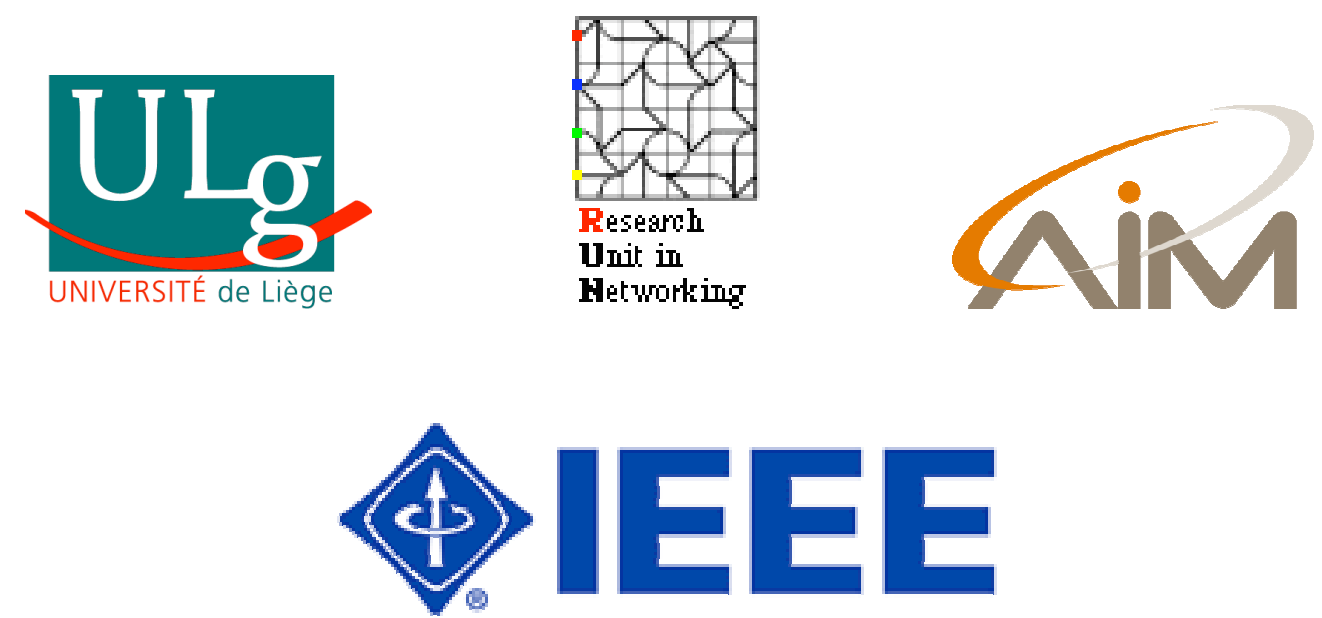